\title{
A hierarchical Bayesian non-linear spatio-temporal model for the spread of invasive species with application to the Eurasian Collared-Dove
}

\author{
Mevin B. Hooten*+ and Christopher K. Wikle*
}

March 27, 2005

\section{Introduction}

Differential equation based advection-diffusion models have long been used in atmospheric science to mimic complex processes such as weather and climate. Differential and partial differential equations (PDE's) have become popular in biological and ecological fields as well. In many cases these models are considered in a strictly deterministic framework even though many sources of uncertainty in the process, the model, and the measurements may exist. Specifically in the ecology realm, Clark et al. 2001 allude to a problem that arises when various sources of uncertainty are not appropriately accounted for. That is, inferences resulting from such methods will be misleading.

The spread of invasive species is one phenomenon in particular that there is much to be (and has already been) learned about through the use of PDE models (Holmes et al. 1994, Shigesada and Kawasaki 2002). Such phenomena usually are based on complicated dynamic processes, many of which are nonlinear and are correlated with and/or controlled by other environmental features (e.g., climate patterns, biotic interactions, physiological characteristics, human population density, etc).

\footnotetext{
${ }^{*}$ Department of Statistics, University of Missouri, Columbia, MO 65211; ${ }^{+}$corresponding author
} 
Many deterministic PDE models are well-equiped to represent the theoretical spread of organisms, but have no mechanism to account for the various sources of uncertainty related to the inadequacies of the model as well as the process itself and our knowledge of it. However the use of a PDE within the framework of a hierarchical Bayesian model can provide a useful link between scientifically based deterministic models and statistical models that accurately portray variability (Wikle 2003).

Specifically, we model the spread of Eurasian Collared-Dove (ECD; Streptopelia decaocto) in the United States using a reaction-diffusion PDE (Fisher 1937, Skellam 1951) within a hierarchical model.

\section{$1.1 \quad$ Background}

The North American Breeding Bird Survey (BBS; Robbins et al. 1986) monitors many birds across the United States and Canada, and provides a major source of data for studying invasive bird species. One such species is the Eurasian Collared-Dove. The ECD is an often a misidentified species in North America (due to its similarity to the Ringed Turtle-Dove) and is considered invasive and a potential threat to indigenous ecosystems (Hengeveld 1993). After moving into Europe in the 1930's, the ECD was introduced in the Bahamas and was observed in the mid-1980's in Florida (Hudson 1965, Romagosa and Labisky 2000). Not only has its range increased as it has spread through the Southeastern United States (Figure 1), but it has experienced growth in population as well (Figure 2).

It should be noted that these data are subject to various types of uncertainty, including observer bias, spatial location error, and error related to change of spatial support (Sauer et al. 1994). Additionally, Hengeveld (1993) and Romagosa and Labisky (2000) suggest that the rate of ECD spread may vary spatially and temporally and may be correlated with other environmental covariates (e.g., human population density). Furthermore, the ECD is an important candidate for study in the United States because of the quantity of data and knowledge accumulated since its introduction combined with historical information gathered from its invasion of Europe 

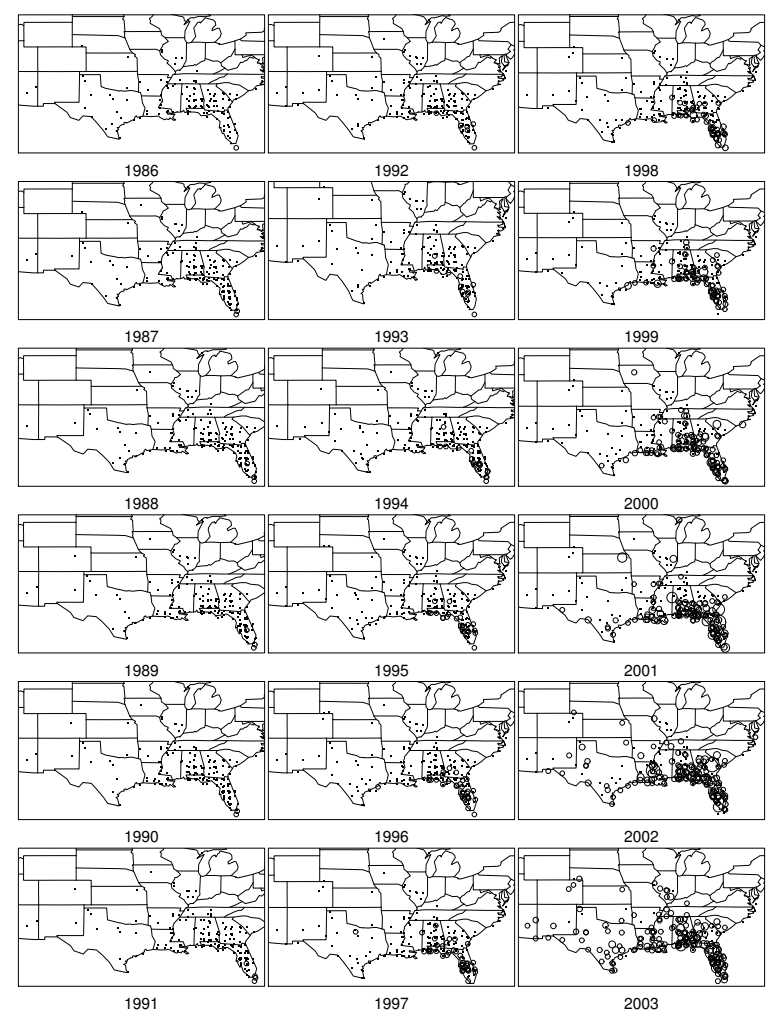

Figure 1: Spread of ECD throughout the United States from 1986 through 2003 (points represent zero counts at sampled location, while circle size corresponds to non-zero count magnitude).

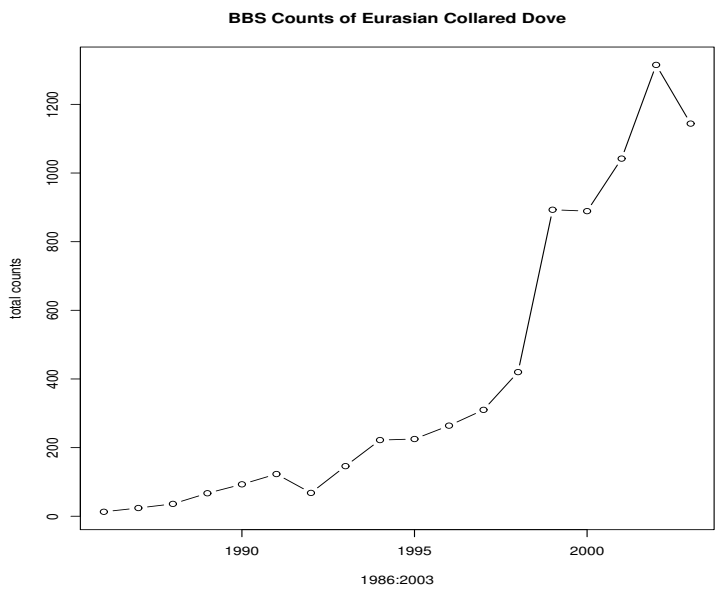

Figure 2: Population growth of ECD in the United States from 1986 through 2003 (total counts over time) 
(Hengeveld 1993).

A model that incorporates these factors and responsibly accounts for the various sources of uncertainty could be a useful tool for ecologists wanting to monitor the spread and growth in population as well as identify factors influencing the rate of spread in order to make management decisions to help protect native species.

\section{Methods}

In order to account for the many sources of uncertainty, spatial effects, temporal effects, population growth, and spread of the invasive species we adopt a 3 -stage hierarchical model that has the following factorization (Berliner 1996): [data, process, parameters $] \propto[$ data $\mid$ process, parameters][process $\mid$ parameters $][$ parameters] (where the square bracket notation refers to distribution).

\subsection{Data Model}

We consider the relative abundances of birds $(n)$ at each spatial location $\left(s_{i}\right)$ and time $(t)$ given an intensity process as Poisson where $m$ is the number of spatial locations and $T$ the number of years:

$$
n_{i, t} \mid \lambda_{t}\left(s_{i}\right) \sim \operatorname{Pois}\left(\lambda_{t}\left(s_{i}\right)\right), \quad i=1, \ldots, m \quad t=1, \ldots, T
$$

In this case, the counts are originally recorded as observed along routes in the spatial domain. For our puposes, the route center is used as the point location for count data $\left(s_{i}\right)$. Since we are concerned about the process on the continental scale, route length (tens of kilometers) and orientation have minimal overall influence.

\subsection{Process Model}

The Poisson intensity process is controlled by a latent spatio-temporal process $\left(\mathbf{u}_{t}\right)$ on a grid linked by an incidence matrix $\left(K_{t}\right)$ that associates observations to grid locations. The number of grid cells (i.e., $\operatorname{dim}\left(\mathbf{u}_{t}\right)=N$ ) need not equal the number of observation locations; in this case $N<m$ increases computational efficiency. We note 
that there may be some consequences related to the effect of grid cell size on parameter estimates. Such associations could be linked to differing biological processes at various scales but will be left as the focus of another study. We use an additive independent noise term to account for observer bias and small scale spatio-temporal variability:

$$
\log \left(\boldsymbol{\lambda}_{t}\right)=K_{t} \mathbf{u}_{t}+\boldsymbol{\varepsilon}_{t}, \quad \boldsymbol{\varepsilon}_{\boldsymbol{t}} \sim N\left(0, \sigma_{\varepsilon}^{2} I\right), \quad t=1, \ldots, T
$$

The latent process $\left(\mathbf{u}_{t}\right)$ is modeled via a reaction-diffusion equation (Fisher 1937, Skellam 1951),

$$
\frac{\partial u}{\partial t}=\frac{\partial}{\partial x}\left(\delta(x, y) \frac{\partial u}{\partial x}\right)+\frac{\partial}{\partial y}\left(\delta(x, y) \frac{\partial u}{\partial y}\right)+\gamma_{0} u\left(1-\frac{u}{\gamma_{1}}\right)
$$

which can be discretized as (Haberman 1987),

$$
\begin{aligned}
u_{t}(x, y)= & u_{t-\Delta_{t}}(x, y)\left[1-2 \delta(x, y)\left(\frac{\Delta_{t}}{\Delta_{x}^{2}}+\frac{\Delta_{t}}{\Delta_{y}^{2}}\right)\right] \\
& +u_{t-\Delta_{t}}\left(x-\Delta_{x}, y\right)\left[\frac{\Delta_{t}}{\Delta_{x}^{2}}\left\{\delta(x, y)-\left(\delta\left(x+\Delta_{x}, y\right)-\delta\left(x-\Delta_{x}, y\right)\right) / 4\right\}\right] \\
& +u_{t-\Delta_{t}}\left(x+\Delta_{x}, y\right)\left[\frac{\Delta_{t}}{\Delta_{x}^{2}}\left\{\delta(x, y)+\left(\delta\left(x+\Delta_{x}, y\right)-\delta\left(x-\Delta_{x}, y\right)\right) / 4\right\}\right] \\
& +u_{t-\Delta_{t}}\left(x, y+\Delta_{y}\right)\left[\frac{\Delta_{t}}{\Delta_{y}^{2}}\left\{\delta(x, y)+\left(\delta\left(x, y+\Delta_{y}\right)-\delta\left(x, y-\Delta_{y}\right)\right) / 4\right\}\right] \\
& +u_{t-\Delta_{t}}\left(x, y-\Delta_{y}\right)\left[\frac{\Delta_{t}}{\Delta_{y}^{2}}\left\{\delta(x, y)-\left(\delta\left(x, y+\Delta_{y}\right)-\delta\left(x, y-\Delta_{y}\right)\right) / 4\right\}\right] \\
& +u_{t-\Delta_{t}}(x, y) \gamma_{0}-u_{t-\Delta_{t}}^{2}(x, y)\left[\frac{\gamma_{0}}{\gamma_{1}}\right] .
\end{aligned}
$$

In matrix notation with additive error, this can be written:

$$
\begin{gathered}
\mathbf{u}_{t}=\mathbf{H}(\boldsymbol{\delta}) \mathbf{u}_{t-1}+\alpha_{0} \mathbf{u}_{t-1}-\alpha_{1} \operatorname{diag}\left(\mathbf{u}_{t-1}\right) \mathbf{u}_{t-1}+\boldsymbol{\eta}_{t} \\
\boldsymbol{\eta}_{t} \sim N\left(0, \sigma_{\eta}^{2} I\right)
\end{gathered}
$$

where $\gamma_{0}$ and $\gamma_{1}$ in (3) represent the intrinsic population growth rate and carrying capacity, respectively, and have the following relationships with the growth parameters 
in the vector form of the model (5):

$$
\alpha_{0}=\gamma_{0}, \quad \alpha_{1}=\frac{\gamma_{0}}{\gamma_{1}}
$$

From (4) we can see where our model differs from that of Wikle (2003). Here the growth in the process is allowed to be non-linear (from the squared term) whereas previously only a linear (Malthusian growth) term was implemented and may not have been appropriate (or sufficient) (Wikle 2003). Although on the log-scale this growth term may not be ideal either, it does allow for a much more flexible and generalized model in that it can always assume the linear form if indeed $\alpha_{1}$ is not significant.

Also, here we consider the discrete $\mathbf{u}$-process on a rectangular grid with spacing $\Delta_{x}$ and $\Delta_{y}$ in the longitudinal and latitudinal directions, respectively, and with time spacing $\Delta_{t}$. The terms $x$ and $y$ refer to the spatial location $(s)$ in two dimensions. The propagator matrix $(H)$ in (5) depends upon the diffusion coefficients $\delta$ which are allowed to vary in space. Construction of $H$ depends on the arrangement of grid cells in the spatial domain and is thus quite project specific. For the irregularly shaped spatial domain considered here the construction of $H$ is similar to that used in Wikle and Hooten (2005).

Such a non-linear model (5) is relatively difficult to implement in an MCMC (Markov Chain Monte Carlo) algorithm due to the high dimensional MetropolisHastings updates. Therefore, we consider the following modification (thereby also inducing a 2nd-order Markov structure).

$$
\mathbf{u}_{t}=H(\delta) \mathbf{u}_{t-1}+\alpha_{0} \mathbf{u}_{t-1}-\alpha_{1} \operatorname{diag}\left(\mathbf{u}_{t-1}\right) \mathbf{u}_{t-2}+\boldsymbol{\eta}_{t}
$$

The $\mathbf{u}_{t-2}$ term in (6) allows for a complete derivation of the full-conditional distribution for $\mathbf{u}_{t}$, thereby improving computational efficiency.

\subsection{Parameter Model}

Let $\mathbf{X}$ be an $m \times 2$ covariate matrix made up of an intercept and human population on the gridded spatial domain and let $\boldsymbol{\beta}$ be a 2-dimensional vector of regression 
coefficients with $\mathbf{R}$ a spatial correlation matrix. Then, $\boldsymbol{\delta}$ can be defined by a linear model,

$$
\boldsymbol{\delta}=X \boldsymbol{\beta}+\boldsymbol{\xi}, \quad \boldsymbol{\xi} \sim N\left(\mathbf{0}, \sigma_{\delta}^{2} \mathbf{R}(\theta)\right)
$$

Let the spatial covariance matrix $(\mathbf{R})$ be defined by the distance $(\|d\|$; the Euclidian distance between points) and the spatial range parameter $(\theta)$ in the standard stationary and isotropic exponential covariogram model:

$$
R(\theta, d)=\exp (-\theta\|d\|)
$$

This allows $\boldsymbol{\delta}$ to utilize not only the human population covariate but the (random) effects of another spatial covariate possibly based on an unknown environmental factor. Also, we let the ECD population growth parameters have a lognormal distribution :

$$
\log (\boldsymbol{\alpha}) \equiv \log \left(\left[\begin{array}{l}
\alpha_{0} \\
\alpha_{1}
\end{array}\right]\right) \sim N\left(\tilde{\boldsymbol{\alpha}}, \Sigma_{\alpha}\right)
$$

The remaining parameters have the following priors:

$$
\begin{aligned}
\boldsymbol{\beta} & \sim N\left(\boldsymbol{\beta}_{0}, \Sigma_{\beta}\right), \\
\sigma_{\varepsilon}^{2} & \sim I G\left(q_{\varepsilon}, r_{\varepsilon}\right), \\
\sigma_{\eta}^{2} & \sim I G\left(q_{\eta}, r_{\eta}\right), \text { and } \\
\sigma_{\delta}^{2} & \sim I G\left(q_{\delta}, r_{\delta}\right) .
\end{aligned}
$$

Additionally we need a prior distribution for the $\mathbf{u}_{t}$-process at time zero (i.e., 1985), thus we specify $\mathbf{u}_{0} \sim N\left(\tilde{\mathbf{u}}_{0}, \Sigma_{0}\right)$. Finally, we adopt a reference prior for the spatial parameter $\theta$ as derived by Berger et al. (2002). Such a reference prior is non-informative and more importantly allows the posterior to be proper. Prior distributions (9-13) were chosen to ensure model conjugacy for purposes of computational and analytical efficiency, while hyperparameters were either chosen to ensure vague priors or, if sufficient information was available, based on previous studies (e.g., rates of ECD spread in Europe). Table 1 shows the numerical values used for the hyperparameters. 
Table 1: Hyperparameters used in MCMC

\begin{tabular}{ll}
\hline Hyperparameter & Value \\
\hline$q_{\varepsilon}$ & 2.3 \\
$r_{\varepsilon}$ & 0.4 \\
$q_{\eta}$ & 2.0 \\
$r_{\eta}$ & 2.0 \\
$q_{\delta}$ & 2 \\
$r_{\delta}$ & 1000 \\
& \\
$\alpha_{0}, \alpha_{1}$ & 0.001 \\
$\left(\Sigma_{\alpha}\right)_{11},\left(\Sigma_{\alpha}\right)_{22}$ & 10 \\
$\left(\Sigma_{\alpha}\right)_{12},\left(\Sigma_{\alpha}\right)_{21}$ & 2 \\
& \\
$\tilde{\mathbf{u}}_{0}$ & $\mathbf{0}$ \\
$\Sigma_{0}$ & $10 \times \mathbf{I}$ \\
\hline
\end{tabular}

The Bayesian formulation of the hierarchical model is summarized by the following posterior distribution and does not have an analytical representation. Therefore, a Gibbs sampler was used to sample from the posterior using the relevant fullconditional distributions.

$$
\begin{aligned}
& {\left[\boldsymbol{\lambda}_{1}, \ldots, \boldsymbol{\lambda}_{T}, \mathbf{u}_{0}, \ldots, \mathbf{u}_{T}, \boldsymbol{\delta}, \boldsymbol{\alpha}, \sigma_{\epsilon}^{2}, \sigma_{\eta}^{2}, \sigma_{\delta}^{2}, \sigma_{\alpha}^{2}, \theta \mid \mathbf{n}_{1}, \ldots, \mathbf{n}_{T}\right]} \\
& \propto \quad\left\{\prod_{t=1}^{T}\left[\mathbf{n}_{t} \mid \boldsymbol{\lambda}_{t}\right]\left[\boldsymbol{\lambda}_{t} \mid \mathbf{u}_{t}, \sigma_{\epsilon}^{2}\right]\right\}\left\{\prod_{t=1}^{T}\left[\mathbf{u}_{t} \mid \boldsymbol{\delta}, \mathbf{u}_{t-1}, \sigma_{\eta}^{2}\right]\left[\mathbf{u}_{0}\right]\right\} \\
& \quad \times \quad\left[\boldsymbol{\delta} \mid \boldsymbol{\alpha}, \sigma_{\delta}^{2}, \theta\right][\boldsymbol{\alpha}]\left[\sigma_{\epsilon}^{2}\right]\left[\sigma_{\eta}^{2}\right]\left[\sigma_{\delta}^{2}\right]\left[\sigma_{\alpha}^{2}\right][\theta]
\end{aligned}
$$

Additionally, non-conjugate parameters $\lambda$ and $\theta$, are sampled via MetropolisHastings updates within the Gibbs sampler.

\section{Results}

This section contains results of the MCMC output and analysis; a discussion of the results follows in the next section. The Gibbs sampler was run for 200,000 iterations 
with a burn-in of 20,000 iterations to ensure convergence of the parameter chains. We can visualize the marginal posterior distribution of model parameters by viewing the histograms constructed from the MCMC samples (Figure 3).
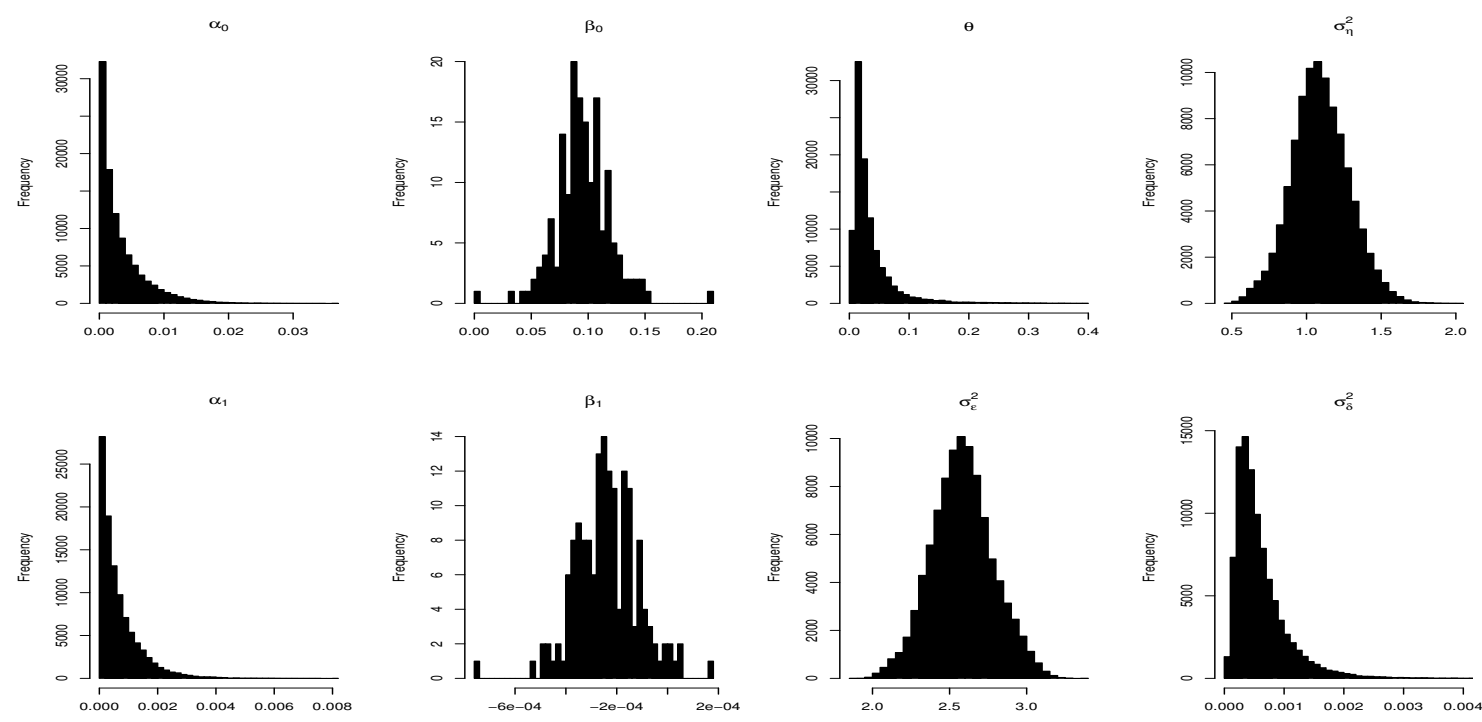

Figure 3: Posterior distributions of univariate $\left(\sigma_{\varepsilon}^{2}, \sigma_{\eta}^{2}, \sigma_{\xi}^{2}, \theta\right)$ and bivariate parameters $(\boldsymbol{\beta}, \boldsymbol{\alpha})$.

Figure 4 shows the posterior mean and standard deviation of the spatial diffusion coefficient $(\boldsymbol{\delta})$ in the form of a map on the spatial domain. The human population covariate (in $\mathbf{X}$ ) is also shown in Figure 4 for comparison.

One way to view the change over time in the Poisson intensity parameter is to plot the posterior mean and $95 \%$ credible interval at a location of interest. Consider ECD growth at a South Florida location (Figure 5). Additionally, by utilizing the MCMC samples from the spatial parameters $\left(\sigma_{\delta}^{2}, \theta\right)$, we can construct a posterior covariogram (assuming the covariogram model in (8)) with $95 \%$ credible interval to give us some idea of the spatial structure in $\delta$ beyond that available in the covariate matrix $(X)$ (Figure 5). We can also view these intensity parameters $(\boldsymbol{\lambda})$ on the spatial domain as a series of maps over time (Figure 6). Additionally, consider the posterior prediction for $\boldsymbol{\lambda}$ (Figure 7). 


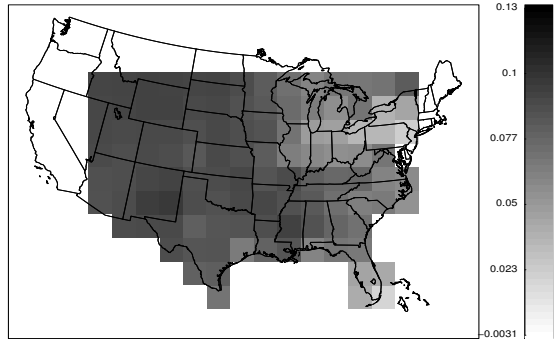

(a) Posterior mean of $\delta$

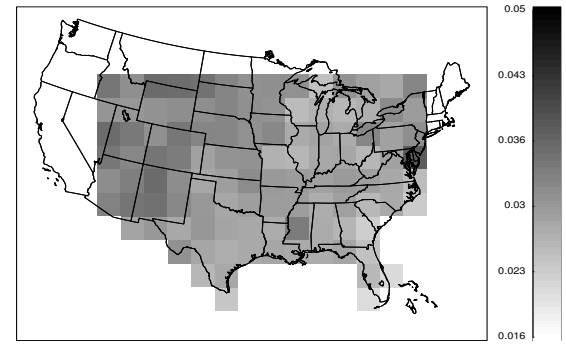

(b) Posterior standard dev. of $\delta$

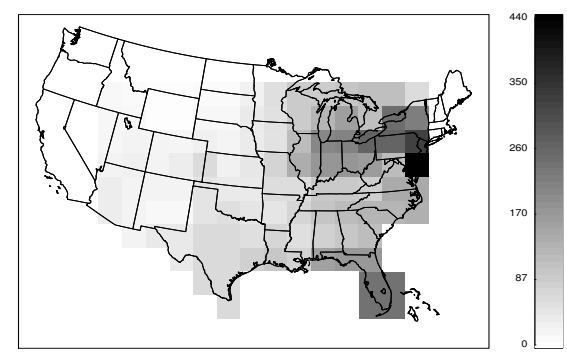

(c) Human population covariate map (i.e., 2nd column of $X$ ).

Figure 4: Posterior summary of $\delta$ and covariate.

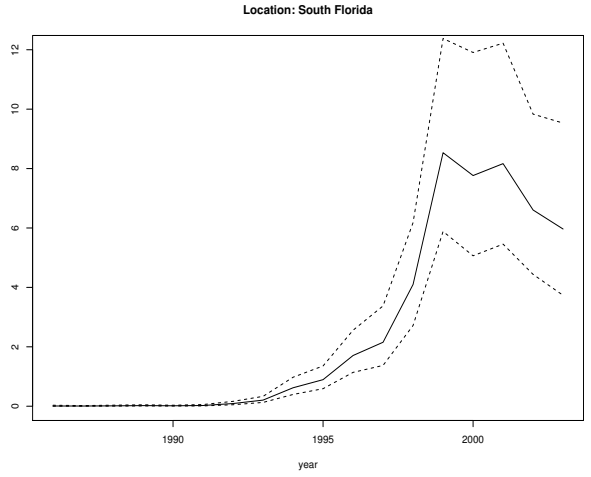

(a) Posterior mean (and 95\% credible interval) of the Poisson intensity process at a South Florida location through time (represents ECD population growth).

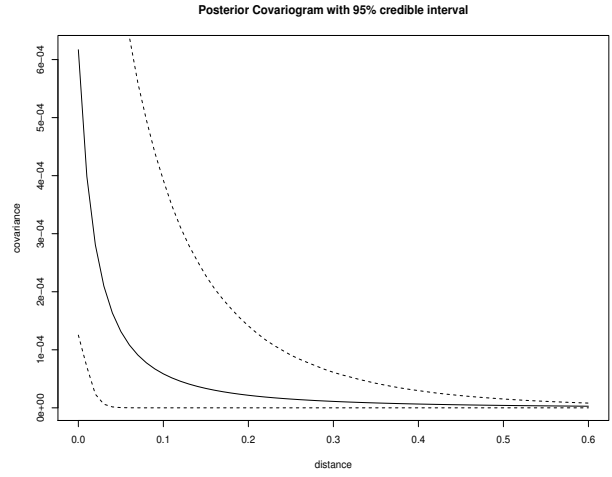

(b) Posterior covariogram with 95\% credible intervals (i.e., covariance structure of $\xi$ ). Maximum distance in the covariogram equals maximum map distance.

Figure 5: Posterior summary of $\lambda$ and $\boldsymbol{\xi}$. 

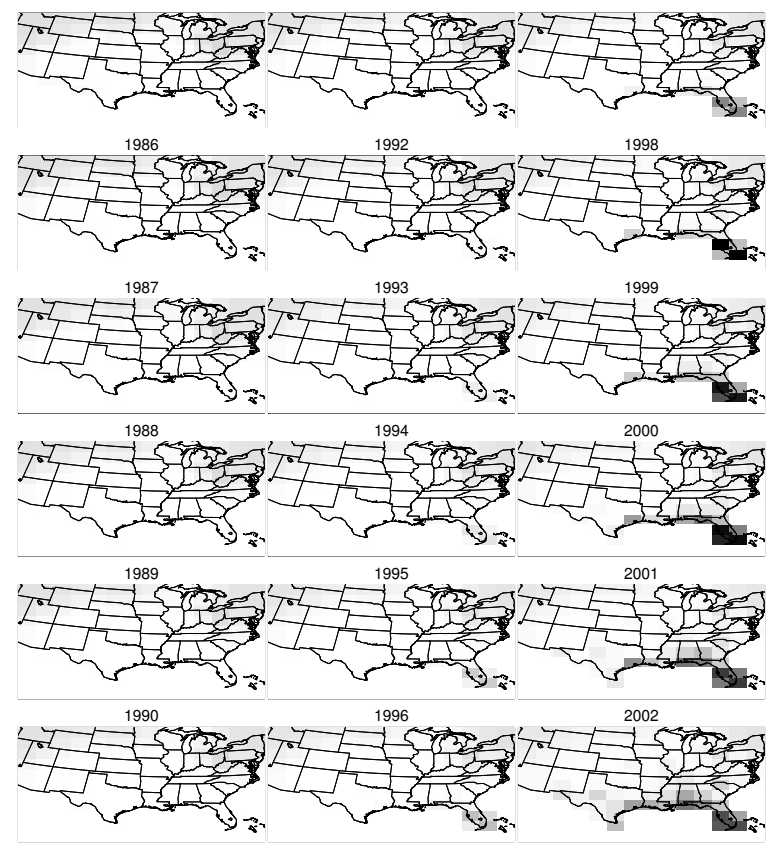

1997

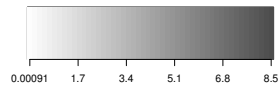

Figure 6: The posterior mean of the Poisson intensity process in time and space.

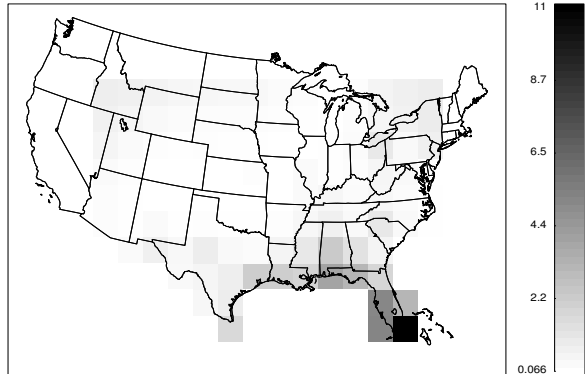

(a) Posterior mean for Poisson intensity prediction.

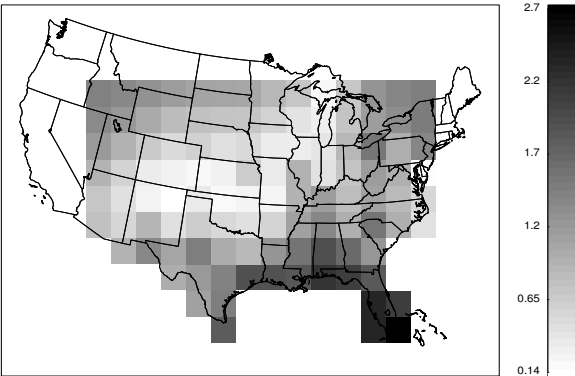

(b) Posterior standard deviation for Poisson intensity prediction.

Figure 7: Posterior prediction of $\boldsymbol{\lambda}$ for 2004. 


\section{Discussion}

It appears that human population density is negatively associated with the rate of diffusion, however the association is only borderline significant (Figures 3, 4), although the intercept coefficient in $\boldsymbol{\beta}$ is clearly significant, suggesting that at least a mean term is important in the model. This goes somewhat against popular scientific opinion, although it could be that ECD relative abundance, rather than rate of diffusion, is more strongly correlated with human proximity. Note also that a few samples of $\delta$ were negative (Figure 4). Technically, $\boldsymbol{\delta}$ should not take negative values, however in this case the model is flexible enough to allow $\delta$ to be negative to make up for other possible deficiencies or misspecifications. Specifically, the human population covariate (Figure 4) contains an outlier and its linear relationship with $\boldsymbol{\delta}$ forces it to be negative. Due to its magnitude, the effect of such an artifact is minimal.

On the other hand, the spatial parameters $\left(\sigma_{\delta}^{2}, \theta\right)$ influencing the varibility in rate of diffusion are significant (Figure 3) suggesting that diffusion is affected by some underlying process (e.g., an unknown latent spatial covariate such as land type, temperature, or precipitation) and are useful in modeling ECD relative abundance in time and space. The covariogram in Figure 5 illustrates that significant spatial structure exists within approximately one third of the maximum map distance; in fact, most (i.e., 95\%) of the spatial structure lies within $1 / 6^{\text {th }}$ of the maximum map distance (i.e., $1 / 6^{\text {th }}$ the width of the United States). This implies that the variability associated with the rate of diffusion that is not attributed to human population is likely due to some unknown spatial covariate with a range of spatial dependence equal to 0.1 in map distance and (or) due to the biological characteristics of ECD dispersal.

The posterior mean (at most locations) of the Poisson intensity process (e.g., Figure 5) shows a brief decrease followed by an increasing trend over time as we would expect and could indeed be the driving force behind the measurements $\left(\mathbf{n}_{t}\right)$. The initial decrease in intensity could be due to an Allee effect (Shigesada and Kawasaki 2002) not present in the original data. Recall that this intensity process is controlled 
by an underlying latent process $\left(\mathbf{u}_{t}\right)$ and thus appears similar (Figure 6) over space and time as $\sigma_{\eta}^{2}$ is quite small and the growth parameters in the PDE are only weakly influential (Figure 3). Moreover, as with similar models (e.g., Wikle 2003), the process is mostly data-driven and the growth component (although possibly misspecified) allows ample flexibility for modeling the relative abundance as well as non-linear population growth and diffusion.

The prediction for the the Poisson intensity process (i.e., mean relative ECD abundance) may be useful for managers wishing to control future range expansion of this species and compare with future monitoring efforts. Notice however that there exists a substantial amount of uncertainty in the prediction (Figure 7), accurate knowledge of which may not be available through other conventional prediction methods.

\section{Conclusions}

The ecology of invasive species is complicated, with non-linear non-seperable spatiotemporal variation influencing the amount of growth and rate of spread of which our knowledge is affected by measurement error and various other sources of uncertainty. We have implemented an ecologically meaningful PDE within a hierarchical Bayesian framework as a latent dynamical system to help manage such uncertainty and account for complicated dependence structures in parameters.

Specifically, inference based on this model suggests that not only does correlation exist between ECD abundance in space and time but the growth and spread of this species across North America can be described and predicted (even at locations without data) while accurately and responsibly accounting for uncertainty. It is important to note that the reaction-diffusion model for the latent process here is one that represents scientific opinion. However, it is by no means the only way to incorporate scientific knowledge into the model. In fact, much more complex hierarchical models could (and should) be considered. 


\section{References}

Berger, J., V. De Oliveira, and B. Sanso. 2002. Objective Bayesian analysis of spatially correlated data. Journal of the American Statistical Association 96:1361-1374.

Berliner, L., 1996. In: Maximum Entropy and Bayesian Methods, Chapter hierarchical Bayesian time series models, pages 15-22. Kluwer Academic Publishers.

Clark, J., S. Carpenter, M. Barber, S. Collins, A. Dobson, J. Foley, D. Lodge, M. Pascual, R. Pielke Jr., W. Pizer, C. Pringle, W. Reid, K. Rose, O. Sala, W. Schlesinger, D. Wall, and D. Wear. 2001. Ecological Forecasts: An emerging imperative. Science 293:657-660.

Fisher, R. 1937. The wave of advance of advantageous genes. Annals of Eugenics 7:355-369.

Haberman, R. 1987. Elementary Applied Partial Differential Equations. Prentice Hall, Inc., Englewood Cliffs, New Jersey, USA.

Hengeveld, R. 1993. What to do about the North American invasion by the Collared Dove? Journal of Field Ornithology 64:477-489.

Holmes, E., M. Lewis, J. Banks, and R. Veit. 1994. Partial differential equations in ecology: Spatial interations and population dynamics. Ecology 75:17-29.

Hudson, R. 1965. The spread of the Collared Dove in Britain and Ireland. British Birds 58:105-139.

Robbins, C., D. Bystrak, and P. Geissler, 1986. The Breeding Bird Survey: its first fifteen years, 1965-1979. Fish and Wildlife Service Resource Publication 157, USDOI, Washington, DC. USA.

Romagosa, C., and R. Labisky. 2000. Establishment and dispersal of the Eurasian Collared-Dove in Florida. Journal of Field Ornithology 71:159-166.

Sauer, J., B. Peterjohn, and W. Link. 1994. Observer differences in the North American Breeding Bird Survey. Auk 111:50-62.

Shigesada, N., and K. Kawasaki, 2002. Dispersal Ecology, Chapter invasion and the long range expansion of species: Effects of long-distance dispersal . Blackwell Publishing, Malden, Massachusetts.

Skellam, J. 1951. Random dispersal in theoretical populations. Biometrika 38:196218.

Wikle, C. 2003. Hierarchical Bayesian methods for predicting the spread of ecological processes. Ecology 84:1382-1394.

Wikle, C., and M. Hooten, 2005. Applications of Computational Statistics in the Environmental Sciences: Hierarchical Bayes and MCMC Methods, Chapter Hierarchical Bayesian spatio-temporal models for population spread, page to appear . Oxford University Press. 